\title{
An introduction to optimal consumption with partial observation
}

\author{
David Lefèvre $^{1} \quad$ Bernt $_{\text {Oksendal }}^{2,3} \quad$ Agnès Sulem $^{1}$
}

November 22, 2000

\begin{abstract}
We give a short introduction to some of the theory and methods involved in stochastic control with partial observation. As an illustration we use the stochastic maximum principle and the Kalman-Bucy filter to solve explicitly a problem about optimal consumption in an economy where the mean relative growth rate is only observed indirectly (partially).
\end{abstract}

\section{Introduction}

Many classic mathematical models in finance assume complete knowledge of all the parameters involved. For example, if an economic quantity $X(t)$ grows like a mean reverting Ornstein-Uhlenbeck process of the form

$$
d X(t)=(\mu-\rho X(t)) d t+\alpha d W(t)
$$

where $\mu, \rho>0$ and $\alpha$ are constants, it is often assumed that these constants are completely observable. However, in practical situations one cannot observe these quantities directly, only indirectly (partially) through the observations of $X(s)$; $s \leq t$. Then the question is: How do we find optimal consumption and/or optimal portfolio in such a situation? This is an example of a stochastic control problem with partial observation.

The purpose of this paper is to give an introduction to the theory and methods of such problems. This is done by studying in detail the specific problem of optimal consumption from the economy (1.1) under a terminal constraint in the case when the mean relative growth rate $\mu$ is only partially observed. We solve this problem explicitly by using the stochastic maximum principle and the Kalman-Bucy filter.

\footnotetext{
${ }^{1}$ INRIA, Domaine de Voluceau-Rocquencourt B.P. 105, F-78153 Le Chesnay Cedex, France email: david.lefevre@inria.fr and Agnes.Sulem@inria.fr

${ }^{2}$ Dept. of Mathematics, University of Oslo, P. O. Box 1053 Blindern, N-0316 Oslo, Norway email: oksendal@math.uio.no

${ }^{3}$ Norwegian School of Economics and Business Administration, Helleveien 30, N-5045 Bergen, Norway
}

Lefèvre, Øksendal \& Sulem: An introduction to optimal ... 
For more information about stochastic control with partial observation we refer to e.g. [B2], [EGK] and the references therein.

\section{Statement of the problem}

\section{a) Complete observation case}

Suppose the wealth $X(t)=X^{(c)}(t)$ of a person with consumption rate $c(t) \geq 0$ satisfies the stochastic differential equation

$$
\left\{\begin{array}{l}
d X(t)=(\mu-\rho X(t)-c(t)) d t+\alpha(t) d W(t) ; t \in[0, T] \\
X(0)=x>0
\end{array}\right.
$$

Here $c(t)=c(t, \omega)$ is an $\mathcal{F}_{t}$-adapted process (our control), $\alpha(t)$ is a given deterministic function and $\mu, \rho, T, x$ are (known) positive constants.

Suppose the expected total discounted utility $J^{(c)}$ corresponding to the consumption rate $c(t)$ is given by

$$
J=J^{(c)}=E\left[\int_{0}^{T} e^{-\delta t} \frac{c^{\gamma}(t)}{\gamma} d t\right]
$$

where $\delta>0, \gamma \in(0,1)$ are (known) constants $(1-\gamma$ is the risk aversion of this person) and $E=E^{x}$ denotes the expectation given that $X(0)=x$.

We say that $c=c(t, \omega)$ is admissible and write $c \in \mathcal{A}$ if $c(t, \omega)$ is $\mathcal{F}_{t}$-adapted, nonnegative and satisfies the terminal condition

$$
E\left[X^{(c)}(T)\right]=x_{T},
$$

where $x_{T} \in \mathbf{R}$ is a given constant. This constraint expresses that on the average a certain wealth $x_{T}$ should be left for the next generation. Consider the problem

Problem 2.1 Find $\bar{J}$ and $\bar{c} \in \mathcal{A}$ such that

$$
\bar{J}:=\sup \left\{J^{(c)} ; c \in \mathcal{A}\right\}=J^{(\bar{c})} .
$$

This is a stochastic control problem with complete information, because we assume that all the parameters involved $(\mu, \rho, \alpha(\cdot), \ldots)$ are known and the consumption rates considered are allowed to be adapted to the filtration $\mathcal{F}_{t}$.

\section{b) Partial observation case}

In the partial observation case the setup and the problem is the same as in (2.1)(2.4) above, but with one important difference: 
We no longer assume that $\mu$ is a known constant. Instead we assume that $\mu=\mu(\omega)$ is a random variable with a known distribution and that $\mu$ is independent of $\{W(s)\}_{s \geq 0}$. Thus the specific value of $\mu$ in (2.1)-(2.4) is not known, and we assume that we cannot observe it directly. We only assume that we can observe it indirectly through the observations of the process $\xi(t)$ defined by

$$
d \xi(t)=\mu d t+\alpha(t) d W(t) ; \quad t \geq 0 \quad \xi(0)=0
$$

We claim that this is equivalent to saying that we observe the no consumption wealth $X^{(0)}(t)$ given by

$$
d X^{(0)}(t)=\left(\mu-\rho X^{(0)}(t)\right) d t+\alpha(t) d W(t) .
$$

To verify this claim, first note that by (2.6), we have

$$
d X^{(0)}(t)+\rho X^{(0)}(t) d t=d \xi(t)
$$

or

$$
d\left(e^{\rho t} X^{(0)}(t)\right)=e^{\rho t} d \xi(t)
$$

which gives

$$
X^{(0)}(t)=X^{(0)}(0) e^{-\rho t}+\int_{0}^{t} e^{-\rho(t-s)} d \xi(s) .
$$

Hence, if we observe $\xi(s) ; s \leq t$ then we know $X^{(0)}(s) ; s \leq t$.

On the other hand, by (2.6) we also have

$$
d \xi(t)=d X^{(0)}(t)+\rho X^{(0)}(t) d t
$$

which gives

$$
\xi(t)=X^{(0)}(t)-X^{(0)}(0)+\rho \int_{0}^{t} X^{(0)}(s) d s .
$$

So, if we know $X^{(0)}(s) ; s \leq t$, we also know $\xi(s) ; s \leq t$. This proves the claim.

Let $\mathcal{G}_{t}$ denote the $\sigma$-algebra generated by $\{\xi(s)\}_{s \leq t}$. We say that $c(t, \omega)$ is admissible for the partial observation problem and write $c \in \mathcal{A}_{p}$ if $c(t, \omega)$ is $\mathcal{G}_{t^{-}}$ adapted, nonnegative and satisfies the terminal condition

$$
E\left[X^{(c)}(T)\right]=x_{T} .
$$

The partial observation problem is

Problem 2.2 Find $J^{*}$ and $c^{*} \in \mathcal{A}_{p}$ such that

$$
J^{*}=\sup \left\{J^{(c)} ; c \in \mathcal{A}_{p}\right\}=J^{\left(c^{*}\right)}
$$

where, as before,

$$
J^{(c)}=E\left[\int_{0}^{T} e^{-\delta t} \frac{c^{\gamma}(t)}{\gamma} d t\right] .
$$

Here $E$ denotes the expectation with respect to the joint probability law of $\mu$ and $\{W(t)\}_{t \in[0, T]}$. 


\section{Optimal consumption with complete observa- tion}

We first solve the complete observation problem (Problem 2.1). To this end we recall the stochastic maximum principle (see e.g. [B1], [P] or [YZ] for proofs and more details):

Suppose $X(t)=X^{(u)}(t)$ is a controlled stochastic system of the form

$$
\left\{\begin{array}{l}
d X(t)=b(t, X(t), u(t) d t+\sigma(t, X(t)) d W(t) ; \quad t \in[0, T] \\
X(0)=x
\end{array}\right.
$$

where $b:[0, T] \times \mathbf{R}^{n} \times U \rightarrow \mathbf{R}^{n}$ and $\sigma:[0, T] \times \mathbf{R}^{n} \rightarrow \mathbf{R}^{n \times m}$ are given $C^{2}$ functions with respect to $x$ whose partial derivatives are uniformly Lipschitz continuous w.r.t. $x$ and uniformly continuous w.r.t. $u$, where $(U, d(\cdot, \cdot))$ is a metric space. $W(t)=\left(W_{1}(t), \ldots, W_{m}(\cdot)\right)^{T}$ is $m$-dimensional Brownian motion and $u:[0, T] \times$ $\Omega \rightarrow U$ is an $\mathcal{F}_{t^{-}}$adapted control process.

Let $f:[0, T] \times \mathbf{R}^{n} \times U \rightarrow \mathbf{R}, g: \mathbf{R}^{n} \rightarrow \mathbf{R}$ and $G: \mathbf{R}^{n} \rightarrow \mathbf{R}^{N}$ be given lower bounded functions satisfying the same conditions as $b$ and $\sigma$ above and define the performance functional

$$
J^{(u)}=E\left[\int_{0}^{T} f(t, X(t), u(t)) d t+g(X(T))\right]
$$

and the terminal condition

$$
E\left[G\left(X^{(u)}(T)\right)\right]=0 .
$$

Let $\mathcal{A}$ denote the set of all $\mathcal{F}_{t}$-adapted processes $u(t, \omega):[0, T] \times \Omega \rightarrow U$ such that $X^{(u)}(t)$ does not explode in $[0, T]$ and such that (3.3) holds. Consider the problem to find $\bar{J} \in \mathbf{R}$ and $\bar{u} \in \mathcal{A}$ such that

$$
\bar{J}=\sup \left\{J^{(u)} ; u \in \mathcal{A}\right\}=J^{(\bar{u})}
$$

If such $\bar{u} \in \mathcal{A}$ exists, then $\bar{u}$ is called an optimal control and $(\bar{u}, \bar{X})$ (where $\bar{X}=$ $\left.X^{(\bar{u})}\right)$ is called an optimal pair.

Define the Hamiltonian $H:[0, T] \times \mathbf{R}^{n} \times U \times \mathbf{R}^{n} \times \mathbf{R}^{n \times m} \rightarrow \mathbf{R}$ by

$$
H(t, x, u, p, q)=b(t, x, u)^{T} p+\operatorname{tr}\left[\sigma(t, x)^{T} q\right]+f(t, x, u)
$$

where $(\cdot)^{T}$ denotes matrix transposed and $\operatorname{tr}[\cdot]$ denotes matrix trace. Consider the following stochastic backward differential equation in the pair of unknown adjoint $\mathcal{F}_{t}$-adapted processes $p(t) \in \mathbf{R}^{n}, q(t) \in \mathbf{R}^{n \times m}$ :

$$
\left\{\begin{array}{l}
d p(t)=-H_{x}(t, X(t), u(t), p(t), q(t)) d t+q(t) d W(t) ; \quad t \in[0, T] \\
p(T)=g_{x}(X(T))+\lambda^{T} G_{x}(X(T))
\end{array}\right.
$$


Here $H_{x}=\nabla_{x} H=\left(\frac{\partial H}{\partial x_{1}}, \ldots, \frac{\partial H}{\partial x_{n}}\right)^{T}$ is the gradient of $H$ with respect to $x$ and similarly with $g_{x}, G_{x} . X(t)=X^{(u)}(t)$ is the process obtained by using the control $u \in \mathcal{A}$ and $\lambda \in \mathbf{R}^{N}$ is a constant. The equation (3.6) is the adjoint equation and $p(t)$ is sometimes interpreted as the shadow price (of a resource).

Theorem 3.1 [The stochastic maximum principle]

a) (Necessary conditions for optimality)

Let $(\bar{u}(t), \bar{X}(t))$ be an optimal pair for problem (3.4). Then there exists a constant $\lambda \in \mathbf{R}^{N}$ and a solution $(p(t), q(t))$ of (3.6) with $u(t)=\bar{u}(t), X=\bar{X}(t)$ such that

$$
\begin{aligned}
& H(t, \bar{X}(t), \bar{u}(t), p(t), q(t)) \\
& \quad=\max _{u \in U} H(t, \bar{X}(t), u, p(t), q(t)) \quad \text { for a.a. }(t, \omega) \in[0, T] \times \Omega .
\end{aligned}
$$

b) (Sufficient conditions for optimality)

Suppose $\lambda \in \mathbf{R}_{+}^{N}, u \in \mathcal{A}$, put $X(t)=X^{(u)}(t)$ and let $p(t), q(t)$ be a solution of the corresponding adjoint equation (3.6). Assume that the functions

$$
H(t, \cdot, \cdot, p(t), q(t)), g(\cdot) \text { and } G(\cdot) \text { are concave for all } t \in[0, T]
$$

and that

$$
\begin{array}{r}
H(t, X(t), u(t), p(t), q(t))=\max _{u \in U} H(t, X(t), u, p(t), q(t)) \\
\text { for a.a. }(t, \omega) \in[0, T] \times \Omega .
\end{array}
$$

Then $(u(t), X(t))$ is an optimal pair for problem (3.4).

For proofs we refer to [B1], [YZ, Chapter 3] and [P].

We now apply the stochastic maximum principle to solve Problem 2.1 (complete observation case).

Here the Hamiltonian function (3.5) is

$$
H(t, x, c, p, q)=(\mu-\rho x-c) p+\alpha(t) q+e^{-\delta t} \frac{c^{\gamma}}{\gamma}
$$

Hence $\frac{\partial H}{\partial x}(t, x, c, p, q)=-\rho p$ and the adjoint equation (3.6) gets the form

$$
\left\{\begin{array}{l}
d p(t)=\rho p(t) d t+q(t) d W(t) ; \quad t \in[0, T] \\
p(T)=\lambda
\end{array}\right.
$$

We immediately see that the solution of this equation is

$$
p(t)=\lambda e^{-\rho(T-t)}, \quad q(t)=0 .
$$


Now $c \rightarrow H(t, x, c, p, q)$ is maximal when

$$
\frac{\partial H}{\partial c}(t, x, c, p, q)=0 \quad \text { i.e. } \quad-p+e^{-\delta t} c^{\gamma-1}=0
$$

This gives

$$
\bar{c}(t)=\left(p(t) e^{\delta t}\right)^{\frac{1}{\gamma-1}}=\lambda^{\frac{1}{\gamma-1}} \exp \left\{\frac{1}{\gamma-1}(-\rho(T-t)+\delta t)\right\}
$$

It remains to find $\lambda$. From (2.1) we have

$$
\left\{\begin{array}{l}
d \bar{X}(t)=(\mu-\rho \bar{X}(t)) d t+\alpha(t) d W(t)-\bar{c}(t) d t ; \quad t \in[0, T] \\
\bar{X}(0)=x
\end{array}\right.
$$

Integrating and taking the expectation we get (using that $\mu$ is constant)

$$
E[\bar{X}(t)]=x+\mu t-\rho \int_{0}^{t} E[\bar{X}(s)] d s-\int_{0}^{t} \bar{c}(s) d s .
$$

Or, with $y(t)=E[\bar{X}(t)]$

$$
y^{\prime}(t)+\rho y(t)=\mu-\bar{c}(t)
$$

which integrates to

$$
e^{\rho T} y(T)=y(0)+\int_{0}^{T} e^{\rho s}(\mu-\bar{c}(s)) d s .
$$

Using the terminal condition (2.3) and (3.13) this gives

$$
\begin{gathered}
x_{T}=x e^{-\rho T}+e^{-\rho T} \int_{0}^{T} e^{\rho s}\left[\mu-\lambda^{\frac{1}{\gamma-1}} \exp \left\{\frac{1}{\gamma-1}(-\rho(T-s)+\delta s)\right\}\right] d s \\
=x e^{-\rho T}+\frac{\mu}{\rho}\left(1-e^{-\rho T}\right)-\frac{1-\gamma}{\delta+\gamma \rho} \lambda^{\frac{1}{\gamma-1}} \exp \left(\frac{\rho \gamma T}{1-\gamma}\right)\left[1-\exp \left(-\frac{\rho+\delta \gamma}{1-\gamma} T\right)\right]
\end{gathered}
$$

Hence,

$$
\begin{array}{r}
\lambda=\left(x e^{-\rho T}+\frac{\mu}{\rho}\left(1-e^{-\rho T}\right)-x_{T}\right)^{\gamma-1}\left[\frac { 1 - \gamma } { \delta + \gamma \rho } \left\{\exp \left(\frac{\gamma \rho T}{1-\gamma}\right)\right.\right. \\
\left.\left.-\exp \left(-\frac{\delta T}{1-\gamma}\right)\right\}\right]^{1-\gamma} \text { if } \delta+\gamma \rho \neq 0 \\
\lambda=\left(x e^{-\rho T}+\frac{\mu}{\rho}\left(1-e^{-\rho T}\right)-x_{T}\right)^{\gamma-1} T^{1-\gamma} \exp (-\delta T) \quad \text { if } \delta+\gamma \rho=0
\end{array}
$$


With this value of $\lambda$ the value of $\bar{c}(t)$ given by (3.13) is optimal, provided that

$$
x e^{-\rho T}+\frac{\mu}{\rho}\left(1-e^{-\rho T}\right) \geq x_{T} .
$$

This condition is necessary to make it possible at all to attain (2.3) for some $u(t) \geq 0$.

We have proved:

Theorem 3.2 Assume that (3.17) holds. Then the optimal consumption rate $\bar{c}(t)$ for the complete observation problem (Problem 2.1) is

$$
\bar{c}(t)=\lambda^{\frac{1}{\gamma-1}} \exp \left\{\frac{1}{\gamma-1}(-\rho(T-t)+\delta t)\right\} ; \quad t \in[0, T]
$$

where $\lambda$ is given by (3.15),(3.16).

\section{Optimal consumption with partial observation}

From now on we assume that

$$
\int_{0}^{T} \alpha^{-2}(t) d t<\infty
$$

Then we have the following fundamental result from linear filtering theory (see e.g. [D], $[\mathrm{Ka}]$ or $[\varnothing])$.

Theorem 4.1 Let $\mathcal{G}_{t}$ be the $\sigma$-algebra generated by $\xi(s), s \leq t$ (see (2.5)). Define the innovation process $B(t)=B(t, \omega)$ by

$$
\left\{\begin{array}{l}
d B(t)=\alpha^{-1}(t)\left(\mu-E\left[\mu \mid \mathcal{G}_{t}\right]\right) d t+d W(t) ; \quad t \geq 0 \\
B(0)=0
\end{array}\right.
$$

Then $B(t)$ is a Brownian motion. Moreover, $B(s) ; s \leq t$ generates the same filtration $\mathcal{F}_{t}$ as $W(s) ; s \leq t$.

Corollary 4.2 With $B(t)$ as in Theorem 4.1 we have

$$
\mu d t+\alpha(t) d W(t)=E\left[\mu \mid \mathcal{G}_{t}\right] d t+\alpha(t) d B(t)
$$

We also need the following result, which is a special case of the Kalman filter formula (see e.g. [D], [Ka] or [Ø, Example 6.2.11]): 
Theorem 4.3 As before let

$$
d \xi(t)=\mu d t+\alpha(t) d W(t)=E\left[\mu \mid \mathcal{G}_{t}\right] d t+\alpha(t) d B(t) ; \quad t \geq 0
$$

and let $\mathcal{G}_{t}$ be the $\sigma$-algebra generated by $\xi(s) ; s \leq t$. Then, if we put

$$
\bar{\mu}=E[\mu] \quad \text { and } \quad A=E\left[(\mu-\bar{\mu})^{2}\right]^{-1}
$$

and

$$
d Y(t)=\alpha^{-2}(t) d \xi(t) \quad \text { and } \quad d Z(t)=\alpha^{-2}(t) d t
$$

we have

$$
\begin{gathered}
E\left[\mu \mid \mathcal{G}_{t}\right]=\left(A+\int_{0}^{t} \alpha^{-2}(s) d s\right)^{-1}\left(\bar{\mu} A+\int_{0}^{t} \alpha^{-2}(s) d \xi(s)\right) \\
=(A+Z(t))^{-1}(\bar{\mu} A+Y(t)) .
\end{gathered}
$$

Using (4.3)-(4.6) we see that we can write our system $X(t)$ in (2.1) on the form

$$
\begin{aligned}
d X(t) & =(-\rho X(t)-c(t)) d t+d \xi(t) \\
& =\left(E\left[\mu \mid \mathcal{G}_{t}\right]-\rho X(t)-c(t)\right) d t+\alpha(t) d B(t) .
\end{aligned}
$$

Note that in this equation all the coefficients are adapted to the observed filtration $\mathcal{G}_{t}$. Thus we have transformed the original partial observation problem (2.1), (2.13) into the complete observation problem (4.7), (2.13). However, the price we pay for this is that the constant $\mu$ in (2.1) is replaced by the more complicated process $E\left[\mu \mid \mathcal{G}_{t}\right]$ in (4.7).

We now proceed to solve the (complete observation) stochastic control problem (4.7), (2.13). To this end, we first write the system in Markovian form, as follows:

$$
\begin{aligned}
& d X(t)=\left[\frac{\bar{\mu} A+Y(t)}{A+Z(t)}-\rho X(t)-c(t)\right] d t+\alpha(t) d B(t) ; \quad X(0)=x \\
& d Y(t)=\alpha^{-2}(t)(A+Z(t))^{-1}(\bar{\mu} A+Y(t)) d t+\alpha^{-1}(t) d B(t) ; Y(0)=y \\
& d Z(t)=\alpha^{-2}(t) d t ; \quad Z(0)=z
\end{aligned}
$$

To emphasize the initial values $x, y, z$ we sometimes write $X^{x}(t), Y^{y}(t)$ and $Z^{z}(t)$ and we let $E^{x, y, z}$ denote the expectation when these initial values are assumed.

In this setting it is natural to extend Problem 2.2 slightly, as follows:

Problem 4.4 Define

$$
J^{(c)}(x)=E^{x, 0,0}\left[\int_{0}^{T} e^{-\delta t} \frac{c^{\gamma}(t)}{\gamma} d t\right] ; \quad s \leq T
$$


Find $J^{*}(x)$ and $c^{*} \in \mathcal{A}_{p}=\mathcal{A}_{p}(x)$ such that

$$
J^{*}(x)=\sup \left\{J^{(c)}(x) ; c \in \mathcal{A}_{p}\right\}=J^{\left(c^{*}\right)}(x) .
$$

To solve this problem we again apply the stochastic maximum principle (Theorem 3.1). In this case the Hamiltonian is

$$
\begin{aligned}
& H(s, x, y, z, c, p, q)=\left(\frac{(\bar{\mu} A+y)}{A+z}-\rho x-c\right) p_{1}+\alpha^{-2}(s)(A+z)^{-1}(\bar{\mu} A+y) p_{2} \\
& \quad+\alpha^{-2}(s) p_{3}+\alpha(s) q_{1}+\alpha^{-1}(s) q_{2}+e^{-\delta s} \frac{c^{\gamma}}{\gamma}
\end{aligned}
$$

where $p=\left(p_{1}, p_{2}, p_{3}\right)^{T}, q=\left(q_{1}, q_{2}, q_{3}\right)^{T}$.

The function $c \rightarrow H(s, x, y, z, c, p, q)$ is maximal when

$$
c=c^{*}(s)=\left(p_{1}(s) e^{\delta s}\right)^{\frac{1}{\gamma-1}} .
$$

The adjoint equation system gets the form

$$
\begin{aligned}
d p_{1}(t) & =\rho p_{1}(t) d t+q_{1}(t) d B(t) \\
d p_{2}(t) & =-\left(A+Z^{0}(t)\right)^{-1}\left[p_{1}(t)+\alpha^{-2}(t) p_{2}(t)\right] d t+q_{2}(t) d B(t) \\
d p_{3}(t) & =\left(A+Z^{0}(t)\right)^{-2}\left[\left(\bar{\mu} A+Y^{0}(t)\right) p_{1}(t)\right. \\
& \left.\quad+\alpha^{-2}(t)\left(\bar{\mu} A+Y^{0}(t)\right) p_{2}(t)\right] d t+q_{3}(t) d B(t)
\end{aligned}
$$

with terminal values

$$
p_{1}(T)=\lambda_{1}, \quad p_{2}(T)=p_{3}(T)=0
$$

where $\lambda_{1} \in \mathbf{R}$ is a constant to be determined.

We see that (4.15) has the solution

$$
p_{1}(t)=\lambda_{1} e^{-\rho(T-t)}, \quad q_{1}(t)=0 .
$$

Hence, by (4.14),

$$
c^{*}(t)=\lambda_{1}^{\frac{1}{\gamma-1}} \exp \left(\frac{1}{1-\gamma}(\rho(T-t)-\delta t)\right) .
$$

To determine $\lambda_{1}$, we now proceed as in the case of complete observations : By integrating the equation

$$
d X^{*}(t)=\left(E\left[\mu \mid \mathcal{G}_{t}\right]-\rho X^{*}(t)-c^{*}(t)\right) d t+\alpha(t) d W(t)
$$

and taking the expectation we get, with

$$
\bar{\mu}=E[\mu]=E\left[E\left[\mu \mid \mathcal{G}_{t}\right]\right],
$$




$$
E\left[X^{*}(t)\right]=x+\bar{\mu} t-\rho \int_{0}^{t} E\left[X^{*}(s)\right] d s-\int_{0}^{t} c^{*}(s) d s .
$$

Hence the terminal condition (2.12) gives, by the same calculation as in Section 3,

$$
\begin{gathered}
\lambda_{1}=\left(x e^{-\rho T}+\frac{\bar{\mu}}{\rho}\left(1-e^{-\rho T}\right)-x_{T}\right)^{\gamma-1}\left[\frac { 1 - \gamma } { \delta + \gamma \rho } \left\{\exp \left(\frac{\gamma \rho T}{1-\gamma}\right)\right.\right. \\
\left.\left.-\exp \left(-\frac{\delta T}{1-\gamma}\right)\right\}\right]^{1-\gamma} \text { if } \delta+\gamma \rho \neq 0 \\
\lambda_{1}=\left(x e^{-\rho T}+\frac{\bar{\mu}}{\rho}\left(1-e^{-\rho T}\right)-x_{T}\right)^{\gamma-1} T^{1-\gamma} \exp (-\delta T) \quad \text { if } \delta+\gamma \rho=0
\end{gathered}
$$

provided that

$$
x e^{-\rho T}+\frac{\bar{\mu}}{\rho}\left(1-e^{-\rho T}\right) \geq x_{T} .
$$

We have proved :

Theorem 4.5 Assume that (4.25) holds. Then the optimal consumption rate $c^{*}(t)$ for the partial observation problem (Problem 2.2) is

$$
c^{*}(t)=\lambda_{1}^{\frac{1}{\gamma-1}} \exp \left(\frac{1}{1-\gamma}(\rho(T-t)-\delta t)\right)
$$

where $\lambda_{1}$ is given by (4.23),(4.24).

Remark 4.6 Note that the solutions $\bar{c}(t)$ and $c^{*}(t)$ of the complete and partial observation problem, respectively, are identical except that the constant $\mu$ in the expression for $\bar{c}(t)$ has been replaced by the constant $\bar{\mu}=E[\mu]$ in the expression for $c^{*}(t)$.

Since $\bar{\mu}$ is assumed known a priori, this means -perhaps surprisingly- that observing $\xi(s) ; s \leq t$ has no effect at all on our consumption rate.

Remark 4.7 In general, we say that a partial observation control problem satisfies the certainty equivalence principle if the following holds : Let $S(\theta)$ denote the optimal control in the complete observation case with known parameter $\theta$. Then $S(\hat{\theta})$ is the optimal control in the partial observation case, where $\hat{\theta}=E\left[\theta \mid \mathcal{G}_{t}\right]$ is the estimate of $\theta$ based on the observation $\mathcal{G}_{t}$.

It is known that the certainty equivalence principle holds in the quadraticlinear partial observation control problems (see e.g. [B2]). On the other hand, it has been proved that in the Merton type of optimal consumption/portfolio problem in a Black-Scholes market with unobservable drift vector, the certainty equivalence principle holds if and only if the utilities are of logarithmic type (see [Ku]). Our problem gives another example where the certainty equivalence principle does not hold. 


\section{The value function}

Finally we find the value functions $\bar{J}=\bar{J}(x)$ and $J^{*}=J^{*}(x)$ of the complete observation problem (Problem 2.1) and the partial observation problem (Problem 2.2), respectively. We could compute $\bar{J}(x)$ and $J^{*}(x)$ by evaluating directly

$$
\bar{J}(x)=E\left[\int_{0}^{T} e^{-\delta t} \frac{\bar{c}^{\gamma}(t)}{\gamma} d t\right] \quad \text { and } \quad J^{*}(x)=E\left[\int_{0}^{T} e^{-\delta t} \frac{\left(c^{*}\right)^{\gamma}(t)}{\gamma} d t\right],
$$

respectively. However, it is easier to use the connection

$$
\frac{d}{d x} \bar{J}(x)=p(0), \quad \frac{d}{d x} J^{*}(x)=p_{1}(0)
$$

between the maximum principle and dynamic programming (see e.g. [YZ, Theorem 5.4.1]):

In the complete observation case this gives, by (3.12)

$$
\frac{d}{d x} \bar{J}(x)=\lambda e^{-\rho T},
$$

where $\lambda=\lambda(x)$ is given by (3.15),(3.16). Therefore

$$
\bar{J}(x)=\frac{1}{\gamma}\left(x e^{-\rho T}+\frac{\mu}{\rho}\left(1-e^{-\rho T}\right)-x_{T}\right)^{\gamma} \psi+C,
$$

where $C$ is a constant and

$$
\psi=\left\{\begin{array}{lll}
{\left[\frac{1-\gamma}{\delta+\gamma \rho}\left\{\exp \left(\frac{\gamma \rho T}{1-\gamma}\right)-\exp \left(-\frac{\delta T}{1-\gamma}\right)\right\}\right]^{1-\gamma} e^{-\rho T}} & \text { if } & \delta+\gamma \rho \neq 0 \\
T^{1-\gamma} \exp (-(\delta+\rho) T) & \text { if } & \delta+\gamma \rho=0
\end{array}\right.
$$

Since $\bar{J}(x)=0$ when $x e^{-\rho T}+\frac{\mu}{\rho}\left(1-e^{-\rho T}\right)=x_{T}$ we have $C=0$. Hence

$$
\bar{J}(x)=\frac{1}{\gamma}\left(x e^{-\rho T}+\frac{\mu}{\rho}\left(1-e^{-\rho T}\right)-x_{T}\right)^{\gamma} \psi .
$$

Similarly, for the partial observation problem we get, by (4.19),

$$
\frac{d}{d x} J^{*}(x)=\lambda_{1}(x) e^{-\rho T}
$$

where $\lambda_{1}=\lambda_{1}(x)$ is given by $(4.23),(4.24)$.

This gives

$$
J^{*}(x)=\frac{1}{\gamma}\left(x e^{-\rho T}+\frac{\bar{\mu}}{\rho}\left(1-e^{-\rho T}\right)-x_{T}\right)^{\gamma} \psi_{1},
$$

where $\psi_{1}=\psi$ is given by (5.3).

We have proved: 
Theorem 5.1 The value functions $\bar{J}(x)$ and $J^{*}(x)$ of the complete observation problem (Problem 2.1) and the partial observation problem (Problem 2.2), respectively, are given by

$$
\begin{aligned}
& \bar{J}(x)=\frac{1}{\gamma}\left(x e^{-\rho T}+\frac{\mu}{\rho}\left(1-e^{-\rho T}\right)-x_{T}\right)^{\gamma} \psi \\
& J^{*}(x)=\frac{1}{\gamma}\left(x e^{-\rho T}+\frac{\bar{\mu}}{\rho}\left(1-e^{-\rho T}\right)-x_{T}\right)^{\gamma} \psi_{1}
\end{aligned}
$$

where $\psi=\psi_{1}$ are both given by (5.3).

\section{Acknowledgements}

This work was partially supported by the French-Norwegian cooperation project Aur 99-050.

\section{References}

[B1] A. Bensoussan: Perturbation Methods in Optimal Control. J. Wiley \& Sons 1988.

[B2] A. Bensoussan: Stochastic Control of Partially Observable Systems. Cambridge Univ. Press 1992.

[D] M.H.A. Davis: Linear Estimation and Stochastic Control. Chapman and Hall 1977.

[EGK] R.J. Elliott, H. Geman and B.M. Korkie: Portfolio optimization and contingent claim pricing with differential information. Stochastics and Stochastics Reports 60 (1997), 185-203.

[Ka] G. Kallianpur: Stochastic Filtering Theory. Springer-Verlag 1980.

[Ku] Y. Kuwana: Certainty equivalence and logarithmic utilities in consumption/investment problems. Math. Finance 5 (1995), 297-309

[Ø] B. Øksendal: Stochastic Differential Equations. Fifth edition. Springer-Verlag 2000.

[P] S. Peng: A general stochastic maximum principle for optimal control problems. SIAM J. Control \& Opt. (1990), 966-979.

[YZ] J. Yong and X.Y. Zhou: Stochastic Controls. Springer-Verlag 1999. 